\title{
INTRODUCING A NEWLY DEVELOPED FABRIC FOR AIR FILTRATION
}

\author{
Mohamed Hakam, Wael Hashima, Ibrahim Elhawary, Adel Elgeiheini \\ Faculty of Engineering, Textile Department, Alexandria University, Alexandria, Egypt \\ E-mail: hakam_tex@yahoo.com
}

\begin{abstract}
:
Woven and nonwoven fabrics present filtration efficiency higher than other air filtration media. Fabrics are selected according to air flow conditions and particle characteristics. The majority of air filtration media are nonwoven fabrics because of their cost, but they need high filtration area for high efficiency. Modified construction of woven fabric introduces high performance in air filtration and decreases filter size, which tends to have better competition abilities. The designed fabrics have considerable thickness and suitable pore characteristics by applying roving instead of weft yarns. Four factors (roving count and their turns per inch, picks per inch and fabric designs) were varied in order to study the effect of these factors on their performance in filtration. Optimum operating conditions for a determined range of air permeability and pore size were obtained.
\end{abstract}

\section{Keywords:}

Roving weft, Air permeability, Pore size, Filtration performance

\section{Introduction}

According to Tharewal et al. [1], in textile industry, the generation of fly and dust affects both laborers' health and product quality. In hospitals, the level of air cleaning and filtration efficiency are very important factors to be controlled. According to Das et al. [2], the various problems of contaminated air cause several types of air filters to be developed. The main objective of air filtration is to remove solid particles from air stream [3-5]. Filters can be used to recover solid particles [1]. Das et al. [2] and Kothari et al. [6] stated that fabric filters, especially woven and nonwoven, are considered the best type of air filtration media. These porous or semi-porous structures with considerable thickness allow air stream to pass through various points of filtration.

According to Irwin [5] and Bird et al. [7], particles bigger than the pore size of filter media are stopped by the membrane. This filtration behavior is called as surface filtration. Many layers of dust accumulate on the filter surface, which increase filtration efficiency. This filtration behavior is called as cake filtration. According to Lawrence et al. [3] and Bird et al. [7], the fabric itself provides the support and true filtering usually occurs through the retained dust cake; this is true for woven filters. Depth filtration occurs whenever the pore size of filtration media is bigger than the particle size. Moreover, some particles block some pores that allow filtration efficiency to increase. This filtration behavior occurs whenever filtration media are thick layers of fibers that are randomly or less orderly arranged like nonwoven filter media. On the other hand, successive blockage during filtration decreases the lifetime of filter. Nonwoven filter media would not be completely cleaned because of the impeded solid particles $[7,8]$.

Air permeability, porosity and pore characteristic affect the performance of air filters [9-11]. Textile materials contain three kinds of pores such as closed pores, blind pores and through pores [12]. Jena and Gupta [13] stated that the largest through pore diameter determines the efficiency of the filtration medium. Maini et al. [14] found that the existence of these pores is a result of the voids within the fiber itself and the voids between the fibers. According to Guangbiao and Fumei [15], inter-yarn porosity has a very strong influence on fabric permeability. Fabric permeability was considered as two parts of inter-yarn and inter-fiber interstices.

According to Tugrul [16], these properties depend mainly on material geometry and construction. Fabric construction, mass per unit area, thickness and packing density are factors controlling air permeability and pore characteristics of woven filters [1, 16]. Modified construction of woven fabric, through replacing the ordinary weft yarns by roving, will increase fabric volume through increasing its thickness. This allows the air stream to path through more and more filtration points. In addition, filtration efficiency is expected to be high with the minimum filtration area.

\section{Materials and methods}

The newly developed fabric was produced using roving as wefts. So, it is very important to produce roving with high turns per inch (TPI) to be inserted easily on the weaving loom. Roving 
with maximum TPI can sustain stresses during insertion as wefts. Roving should be rewind on cone package to be suitable for loom creel. On the rapier loom, the rapier head has the ability to grip and genteelly insert roving across the shed. The fabric constructions consist of constant warp of 20/2 Ne, 13 TPI and 22 ends per inch. Warp and weft have the same material, which consists of $50 \%$ cotton or $50 \%$ cotton waste. The weft yarns were obtained by varying both roving count $(0.8,1,1.2$ $\mathrm{Ne})$ and TPI $(1.4,1.8,2.2)$. Three levels of picks per inch (PPI; $16,20,24)$ and three fabric designs (plain, twill, sateen) were applied for obtaining the modified fabrics. Regression analysis was applied on the obtained results to attain the best operating conditions. Some fabrics of the suitable filtration properties were selected for the design of air filters. Selected fabrics for filter design had the same fabric construction, except weave design, which was as follows: roving count $1 \mathrm{Ne}, 1.4 \mathrm{TPI}$ and $16 \mathrm{PPI}$. The designed filters were subjected to air filtration tests in order to study and evaluate their performance.

\subsection{Fabric testing}

Fabric mass was evaluated according to ASTM D 3776-96 (reapproved 2002). The air permeability test was carried out according to ISO 9237. The maximum pore size was measured according to British standards (BS 3321). Fabric thickness was evaluated according to ASTM standard D 46 (1975). Packing density was calculated based on the results of fabric mess and thickness tests through equation $P_{\mathrm{d}}=1000{ }^{*} M / T$, where $P_{\mathrm{d}}$ is the fabric packing density $\left(\mathrm{gm} / \mathrm{cm}^{3}\right), M$ the fabric mass per square meter $\left(\mathrm{gm} / \mathrm{m}^{2}\right)$ and $T$ the fabric thickness $(\mathrm{mm})$.

\subsection{Filter testing}

Three properties are measured in order to determine the performances of tested filter according to International
Standard ISO 5001/2000 and Egyptian Standard ES 918/2007 $[17,18]$. It is also recommended that the most important properties affecting filtration performance are resistance to air flow, average filtration efficiency and filter dust capacity. The previous properties were measured during the air filtration test, which are classified into three tests.

1. Resistance to air flow test: The test is carried out by the device shown in figure 2.1. Without dust loading and eject the absolute filter which shown in figure 2.2.a and housing 2.2.b [19]. High-efficiency particulate air absolute filter introduces a minimum efficiency of $99.97 \%$ when tested at particles' diameter of $0.3 \mathrm{~mm}$, which is recommended to use in air filtration tests to evaluate and test the performance and filtration efficiency of a new filter before operation. The properties of the used absolute filter are shown in figure 2.2.c. Resistance to the flow test is carried out through operating the system with and without the tested filter and then, the pressure drops $D p_{1}$ and $D p_{2}$ are determined in both cases. Pressure drop of the filter can be calculated by the following equation: $\mathrm{D} p=\mathrm{D} p_{2}-\mathrm{D} p_{1}$. The calculation is repeated with several values of air flow rate, and pressure drop of the filter is determined for each flow rate.

2. Filtration efficiency test: The system is operated for 10 minutes before dust loading to adjust the air flow rate, and then, the dust injector is operated. The filtration efficiency test can be performed as follows: masses of tested and absolute filters are measured before and after the test to determine the dust mass on the tested filter $\left(M_{\mathrm{t}}\right)$ and absolute filter $\left(M_{\mathrm{a}}\right)$. Filtration efficiency can be determined by the following equation:

$$
\% \eta=\frac{M_{\mathrm{t}}}{M_{\mathrm{t}}+M_{\mathrm{a}}} \times 100
$$

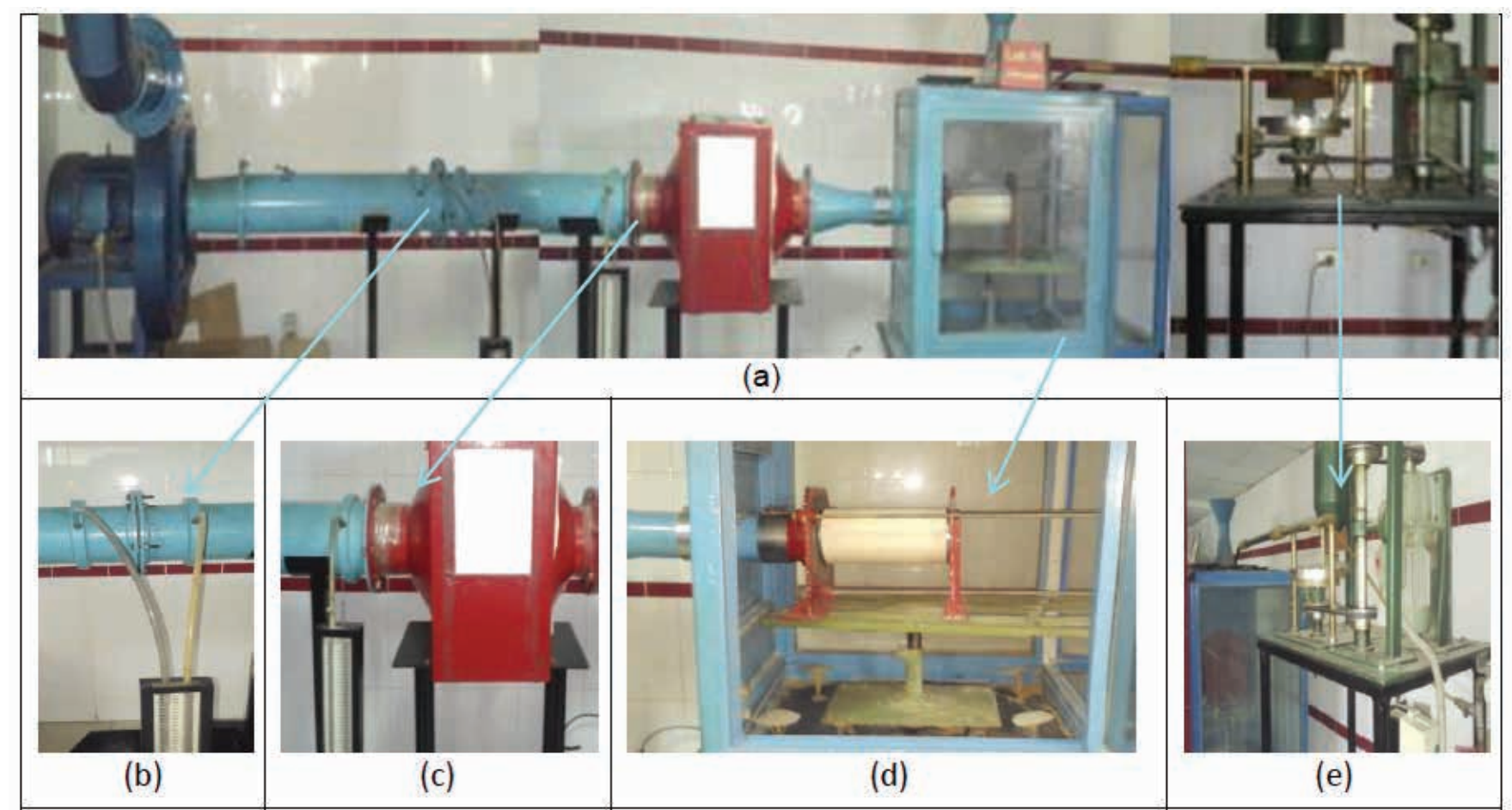

Figure 2.1. (a) The main parts of filtration efficiency device: (b) the first manometer after suction pump, (c) the second manometer and absolute filter housing, (d) inside glass test room and (e) dust feeder (dust injector). 

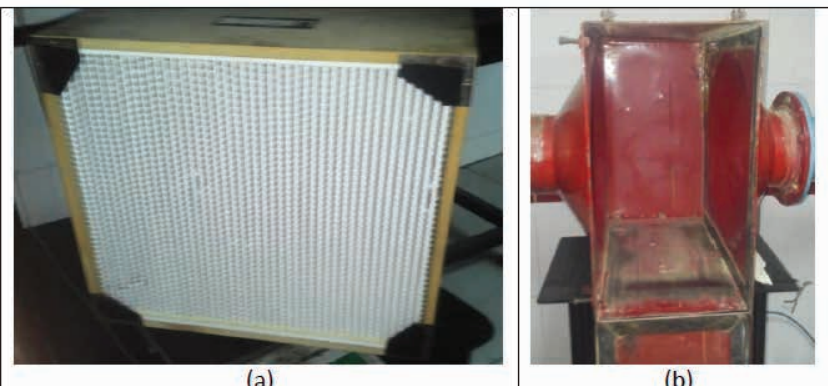

(b)

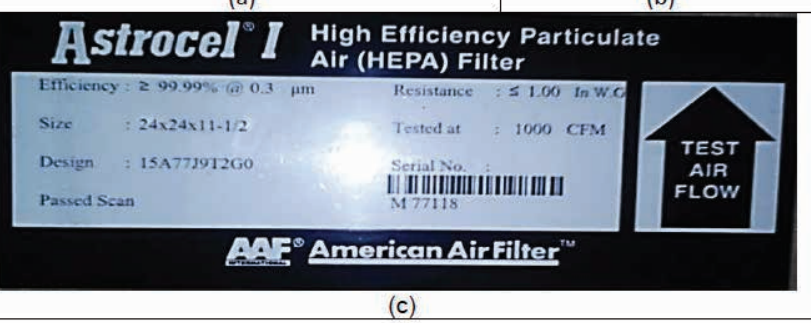

Figure 2.2. The absolute filter construction (a), open housing (b) and its label (c).

3. Dust capacity of a filter: It is defined as the ratio of dust mass accumulated on the tested filter $\left(M_{t}\right)$ to the total dust mass injected during the filtration test. Dust concentration should be within the range ( 1 to $3 \mathrm{gm} / \mathrm{m}^{3}$ ) of air. Dust particles are prepared according to ISO 12103-A4 for the coarse dust test. The dust preparation device mainly consists of five metallic meshes. Table 2.1 shows pore diameter of each mesh, the range of particles' size on its surface and the percentage of sharing in total dust mix.

\section{Results and discussion}

Figure 3.1 shows the structure of the developed fabric. Significant regression equations, concerning the relations between the factors under study and the measured properties, were determined and are represented in table 3.1, which demonstrate that the proposed regression represents significantly the obtained data since $R^{2}$ is higher than 0.91 and the F-significance tends to zero. Also the roving counts, PPI and their interactions are the most important factors affecting all the tested properties. The regressions coefficients in case of air permeability and maximum pore size are the same, with the same sign and rank. The effects of different factors on some properties are discussed according to the experimental data as follows.

\subsection{Fabric air permeability}

Figure 3.2 shows that air permeability increases as roving count increases for minimum and medium PPI. Whenever roving count becomes finer than $1 \mathrm{Ne}$, permeability decreases especially for plain weave of $24 \mathrm{PPI}$. Practically, plain fabric is not woven easily for maximum PPI or maximum cover. So, coarse roving forces weaving loom to decrease the actual PPI in the produced fabric, while fine roving allows the actual PPI to increase and tend to be as the same as the designed 24PPI. However, for coarse counts, the actual PPI is lesser than the designed one. So, air permeability decreases as roving count becomes finer than $1 \mathrm{Ne}$ for maximum PPI. It is normal relationship between roving count and air permeability at 16
Table 2.1. Pore size of each mesh and percentage of sharing in the final dust mix.

\begin{tabular}{|c|c|c|c|}
\hline $\begin{array}{c}\text { Mesh } \\
\text { number }\end{array}$ & $\begin{array}{c}\text { Pore } \\
\text { diameter of } \\
\text { mesh }(\boldsymbol{\mu m})\end{array}$ & $\begin{array}{c}\text { Size of dust } \\
\text { particles }(\boldsymbol{\mu m})\end{array}$ & $\begin{array}{c}\text { Percentage } \\
\text { of sharing in } \\
\text { dust mix (\%) }\end{array}$ \\
\hline 1 & 212 & $\mathrm{D}>212$ & 0 \\
\hline 2 & 180 & $180<\mathrm{D} \leq 212$ & 21 \\
\hline 3 & 75 & $75<\mathrm{D} \leq 180$ & 27 \\
\hline 4 & 38 & $38<\mathrm{D} \leq 75$ & 14 \\
\hline 5 & 25 & $D \leq 25$ & 38 \\
\hline
\end{tabular}
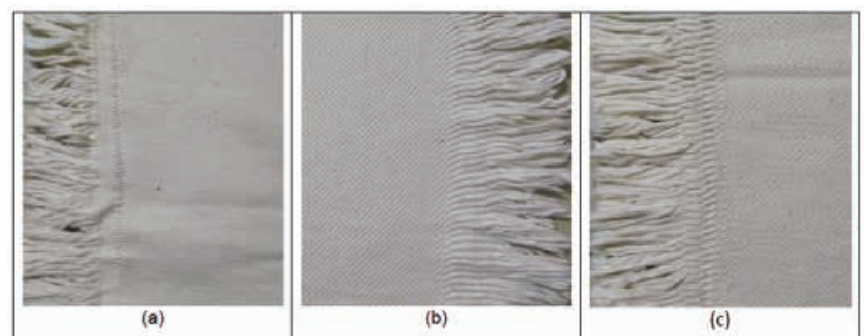

Figure 3.1. Three designs of the produced fabric: (a) plain weave, (b) twill weave and (c) sateen weave.

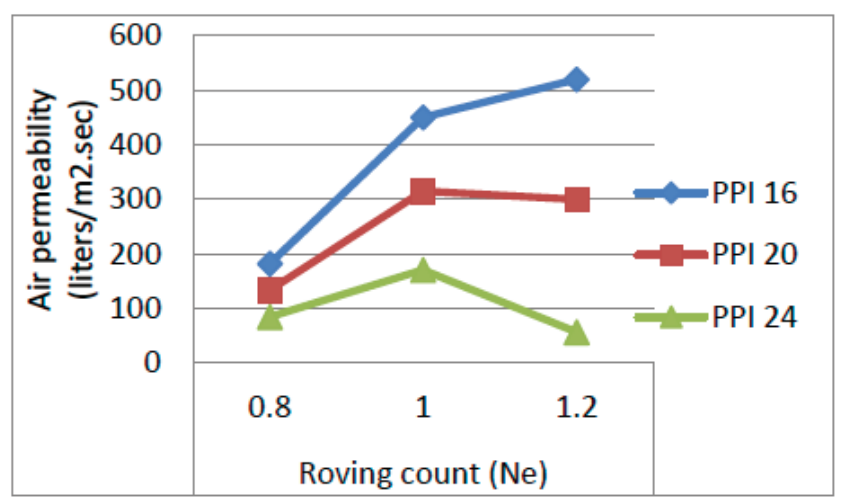

Figure 3.2. Effect of roving count and PPI on fabric permeability for 1.8 weft TPI plain weave.

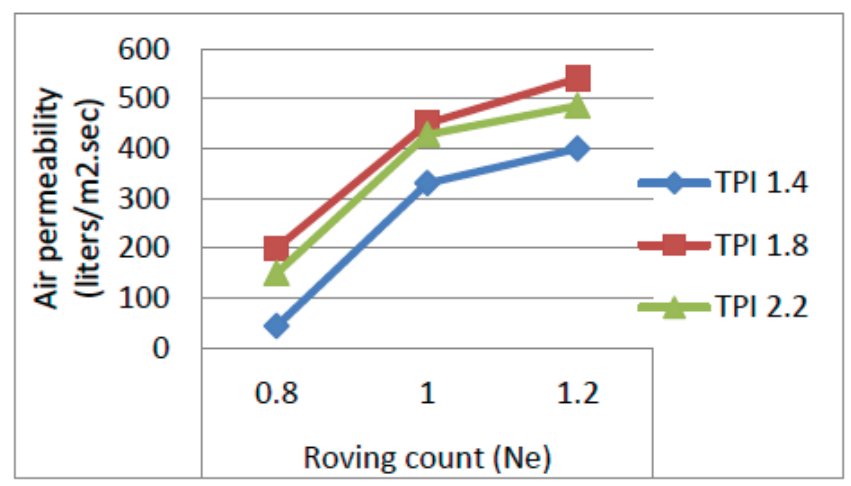

Figure 3.3. Effect of roving count and TPI on fabric permeability for 16 PPI twill weave.

PPI. As shown in figure 3.3, air permeability increases as roving TPI increases up to 1.8 , and then, there is a little reduction in air permeability. The maximum vales of air permeability can be obtained at the medium level of roving TPI. It can be found that the newly developed fabric depends on roving in its structure. Air passes through voids between roving within fabric structure, and also, air can path through pores between 
Table 3.1. Regression equations for measured properties and studied factors.

\begin{tabular}{|c|c|c|c|c|c|c|c|}
\hline & \multirow{2}{*}{ Property } & \multicolumn{2}{|c|}{ Air permeability (liter $/ \mathrm{m}^{2} \times \mathrm{sec}$ ) } & \multicolumn{2}{|c|}{ Maximum pore size $(\mu \mathrm{m})$} & \multicolumn{2}{|c|}{ Packing density $\left(\mathrm{gm} / \mathrm{cm}^{3}\right)$} \\
\hline & & Level & $p$-value & Level & $p$-value & Level & $p$-value \\
\hline \multicolumn{8}{|c|}{ Plain } \\
\hline \multicolumn{2}{|r|}{ Constant } & 319 & $<0.01$ & 174 & $<0.01$ & 0.54 & $<0.01$ \\
\hline \multirow{3}{*}{$\begin{array}{l}\pi \\
\stackrel{0}{0} \\
\frac{0}{0} \\
\frac{0}{\omega}\end{array}$} & $\mathrm{X} 1$ & 74 & $<0.01$ & 36 & $<0.01$ & -0.03 & $<0.01$ \\
\hline & $\mathrm{X} 2$ & 17 & $<0.01$ & 14 & $<0.01$ & 0.02 & 0.03 \\
\hline & X3 & -141 & $<0.01$ & -78 & $<0.01$ & 0.06 & $<0.01$ \\
\hline \multirow{4}{*}{ 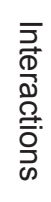 } & $\mathrm{X} 23$ & -34 & $<0.01$ & -15 & $<0.01$ & & \\
\hline & $\mathrm{X} 13$ & -93 & $<0.01$ & -42 & $<0.01$ & -0.03 & 0.01 \\
\hline & $\mathrm{X} 11$ & -99 & $<0.01$ & -37 & $<0.01$ & & \\
\hline & $\mathrm{X} 22$ & -112 & $<0.01$ & -42 & $<0.01$ & -0.03 & 0.03 \\
\hline \multicolumn{2}{|r|}{$\mathrm{R}^{2}$} & \multicolumn{2}{|l|}{0.97} & \multicolumn{2}{|l|}{0.96} & \multicolumn{2}{|l|}{0.96} \\
\hline \multicolumn{2}{|c|}{ Significance F } & \multicolumn{2}{|l|}{ Significant } & \multicolumn{2}{|l|}{ Significant } & \multicolumn{2}{|l|}{ Significant } \\
\hline \multicolumn{8}{|c|}{ Twill } \\
\hline \multicolumn{2}{|r|}{ Constant } & 345 & $<0.01$ & 192 & $<0.01$ & 0.49 & $<0.01$ \\
\hline \multirow{3}{*}{ 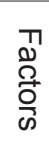 } & $\mathrm{X} 1$ & 76 & $<0.01$ & 39 & $<0.01$ & -0.04 & $<0.01$ \\
\hline & $\mathrm{X} 2$ & 20 & $<0.01$ & 16 & $<0.01$ & 0.14 & 0.07 \\
\hline & X3 & -141 & $<0.01$ & -77 & $<0.01$ & 0.04 & $<0.01$ \\
\hline \multirow{4}{*}{ 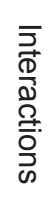 } & $\mathrm{X} 23$ & -34 & $<0.01$ & -16 & $<0.01$ & & \\
\hline & $\mathrm{X} 13$ & -93 & $<0.01$ & -43 & $<0.01$ & & \\
\hline & $\mathrm{X} 11$ & -104 & $<0.01$ & -39 & $<0.01$ & & \\
\hline & $\mathrm{X} 22$ & -117 & $<0.01$ & -40 & $<0.01$ & -0.03 & 0.04 \\
\hline \multicolumn{2}{|r|}{$\mathrm{R}^{2}$} & \multicolumn{2}{|l|}{0.99} & \multicolumn{2}{|l|}{0.95} & \multicolumn{2}{|l|}{0.91} \\
\hline \multicolumn{2}{|c|}{ Significance $\mathrm{F}$} & \multicolumn{2}{|l|}{ Significant } & \multicolumn{2}{|l|}{ Significant } & \multicolumn{2}{|l|}{ Significant } \\
\hline & Sateen & & & & & & \\
\hline & Constant & 347 & $<0.01$ & 210 & $<0.01$ & 0.45 & $<0.01$ \\
\hline & $\mathrm{X} 1$ & 86 & $<0.01$ & 37 & $<0.01$ & -0.05 & $<0.01$ \\
\hline O⿱艹 & $\mathrm{X} 2$ & & & 11 & $<0.01$ & & \\
\hline & X3 & -150 & $<0.01$ & -82 & $<0.01$ & 0.02 & 0.04 \\
\hline & $\mathrm{X} 23$ & -34 & $<0.01$ & -14 & $<0.01$ & & \\
\hline$\frac{\overrightarrow{\mathbb{D}}}{\mathbb{Q}}$ & $\mathrm{X} 13$ & -72 & $<0.01$ & -40 & $<0.01$ & & \\
\hline$\frac{9}{0}$ & $\mathrm{X} 11$ & -106 & $<0.01$ & -39 & $<0.01$ & & \\
\hline$\vec{\omega}$ & $\mathrm{X} 22$ & -98 & $<0.01$ & -43 & $<0.01$ & & \\
\hline & $\mathrm{R}^{2}$ & 0.96 & & 0.96 & & 0.79 & \\
\hline & VIFICANCE $F$ & SIGNIFICA & & SIGNIFICA & & SIGNIFIC & \\
\hline
\end{tabular}

X1, weft count Ne; X2, weft turns per inch; X3, picks per inch.

fibers within roving structure. Roving diameter decreases as its TPI increases. So, voids or spaces between roving within the weave structure have to be enlarged. However, TPI higher than 1.8 decreases the voids between fibers within the roving structure and decreases the resultant fabric permeability. Fabric permeability can be controlled by controlling twist level for the same roving count.

\subsection{Fabric pore size}

As stated the effect of studied factors on pore size is typically as that on air permeability. It can be observed from figure 3.4 that pore size increases as roving TPI increases up to 1.8 TPI and then pore size decreases, which is explained previously at air permeability discussion. The figure shows that minimum values of pore size can be obtained at plain weave. Figure 3.5 shows that the rate of reduction in pore size due to increasing roving TPI, more than 1.8, in twill weave is lower than that of plain weave. This rate of reduction is the least for sateen weave, which is considered to introduce soft fabric and is easily woven with high PPI, as shown in figure 3.6. Increasing roving count results in increasing pore size in the fabric produced of 16 and $20 \mathrm{PPI}$. There is a small reduction in the pore size for the fabric of $24 \mathrm{PPI}$, which is discussed previously at air permeability discussion. Since air permeability and pore size are geometrical 


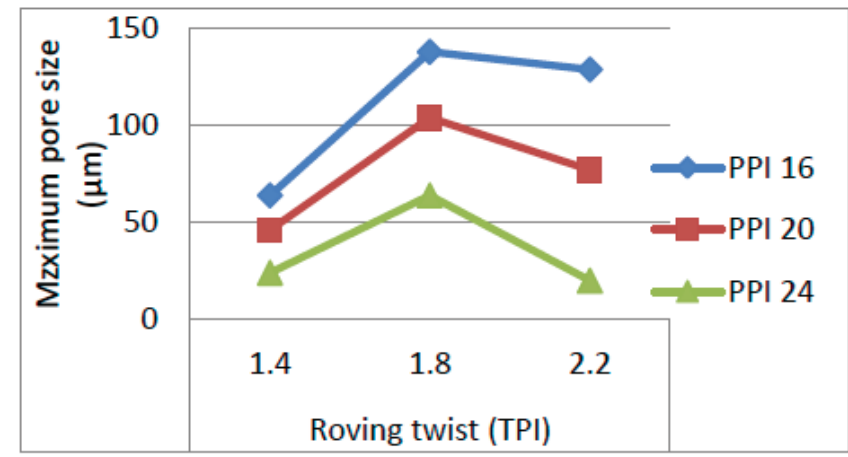

Figure 3.4. Effect of roving TPI and PPI on pore size for $0.8 \mathrm{Ne}$ weft plain weave.

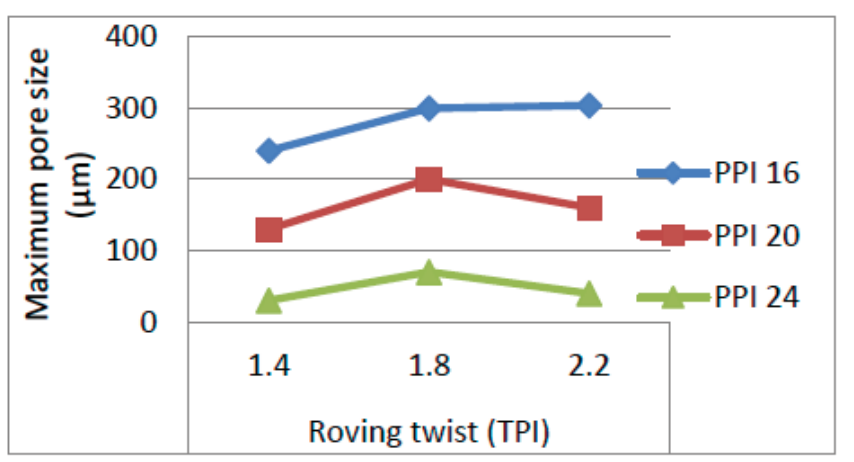

Figure 3.5. Effect of roving TPI and PPI on pore size for $1.2 \mathrm{Ne}$ weft twill weave.

properties of a material, so yarn or roving structures, count, PPI, TPI and fabric design affect these properties.

\subsection{Fabric packing density}

Packing density is determined based on results of both fabric mass and thickness tests. As stated before, packing density a (mass/thickness), for the same fabric area. As shown in figure 3.7, packing density increases as roving TPI increases for the medium $20 \mathrm{PPI}$. It can be explained that increasing TPI results in decreasing roving diameter, which decreases fabric thickness, and packing density increases as the mass remains constant. At $24 \mathrm{PPI}$, whenever roving TPI increases, also diameter reduces, which decreases both fabric thickness and its mass. It can be explained that the actual PPI in the produced fabric tends to be as the same as the designed PPI for the finer roving with a lower diameter to give the same fabric cover. Then, fabric mass tends to increase as actual PPI in the fabric increases. So, the resultant packing density remains constant in this case. As shown in figure 3.8, the finer the count, the lower the packing density. At 1.8 roving TPI, relations between measured properties and studied factors will be normal. The rate of reduction in packing density for $24 \mathrm{PPI}$ is higher than that for $16 \mathrm{PPI}$, which is discussed previously. The maximum PPI in the produced fabric needs roving with a low diameter to be woven easily. Finer roving means lower diameter, and actual PPI increases. In general, sateen weave has the least packing density than twill or plain weaves. Sateen weave is much thicker than other designs, plain and twill, according to the results of the fabric thickness test. So, sateen is more permeable than twill or plain weaves because of its low packing density.

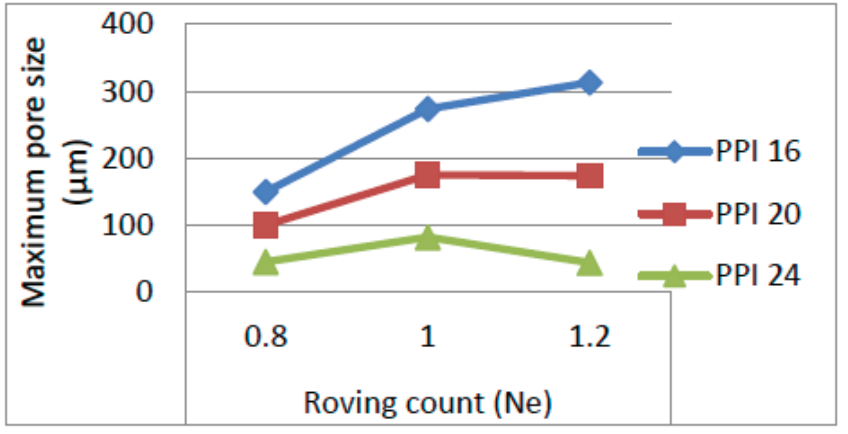

Figure 3.6. Effect of roving count and PPI on pore size for 2.2 weft TPI sateen weave.

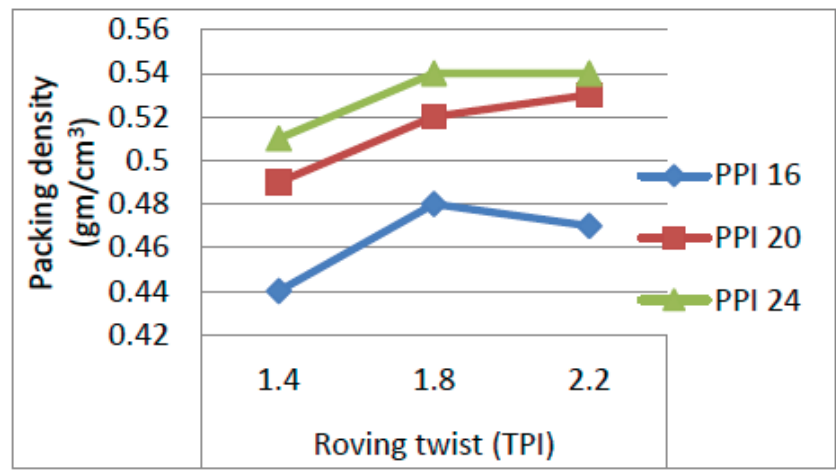

Figure 3.7. Effect of roving TPI and PPI on packing density for $0.8 \mathrm{Ne}$ weft plain weave.

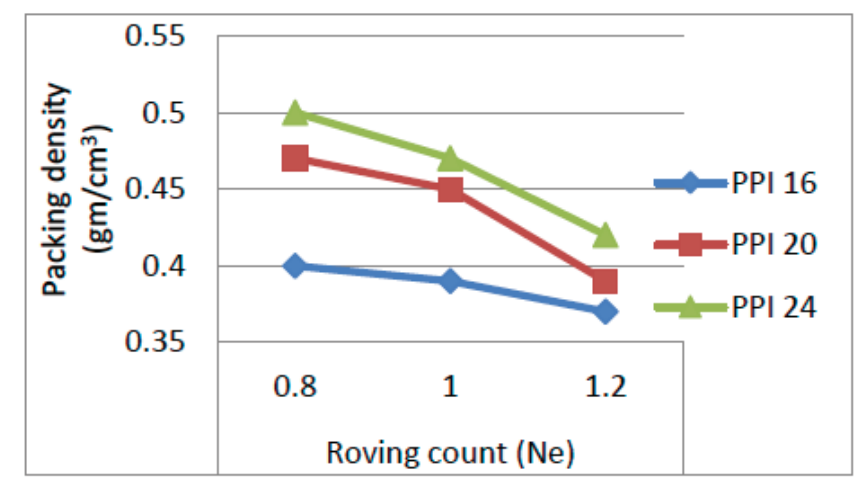

Figure 3.8. Effect of roving count and PPI on packing density for 1.8 weft TPI sateen weave.

\subsection{Filtration performance of the designed filters}

Figure 3.9 shows the designed filters that were prepared for the air filtration test. This type of filters, which is known as surface filter, needs area of filtration media lower than that for nonwoven pocket filters, which consume large area of fabric. The performance of the designed filtration media was evaluated as follows.

\subsubsection{Resistance to air flow}

Figure 3.10 illustrates the relation between air flow rate and pressure drop for a plain filter. The figure presents that filter's pressure drop increases rapidly as the air flow rate increases from 3.3 to $3.5 \mathrm{~m}^{3} / \mathrm{min}$. Within this range, it can be noticed that pressure drop increases by $40 \%$ as the air flow rate increases by $6 \%$. The pressure drop increases slightly whenever the air 


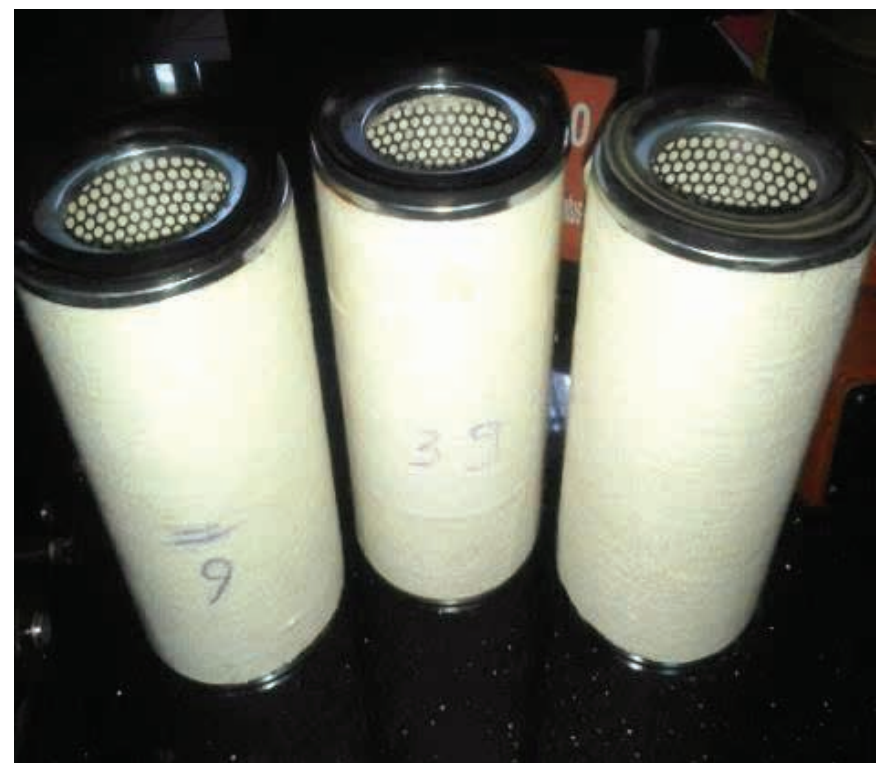

Figure 3.9. The designed air filters.

flow rate increases from 3.5 to $4.1 \mathrm{~m}^{3} / \mathrm{min}$. For this filter, the optimum filtration performance can be achieved whenever the air flow rate varies between 3.5 and $4.1 \mathrm{~m}^{3} / \mathrm{min}$. Within the previous range of air flow rate, it can be noticed that pressure drop increases by $13 \%$ as the air flow rate increases by $20 \%$. So, the air flow rate has little effect on the pressure drop of a filter within this range of flow.

Figure 3.11 demonstrates the relation between air flow rate and pressure drop for a twill filter. The figure shows that filter pressure drop increases rapidly as the air flow rate increases from 3.6 to $4.1 \mathrm{~m}^{3} / \mathrm{min}$. Within the previous range of air flow rate, it can be noticed that pressure drop increases by $39 \%$ as the air flow rate increases by $14 \%$. The pressure drop increases slightly whenever the air flow rate increases from 4.1 to $4.8 \mathrm{~m}^{3 /}$ $\mathrm{min}$. It is recommended for this filter that the optimum filtration performance can be achieved whenever the air flow rate varies between 4.1 and $4.8 \mathrm{~m}^{3} / \mathrm{min}$. Within the previous range of air flow rate, it can be noticed that pressure drop increase by $9 \%$ as the air flow rate increases by $17 \%$. So, the air flow rate has a little effect on the pressure drop of a filter within this range of flow.

Figure 3.12 shows the relation between air flow rate and pressure drop for a sateen filter of $1 \mathrm{Ne}$ weft with $1.4 \mathrm{TPI}$ and 16 PPI. The figure introduces that filter's pressure drop increases rapidly as the air flow rate increases from 4 to $5.3 \mathrm{~m}^{3} / \mathrm{min}$. Within the previous range of air flow rate, it can be noticed that pressure drop increases by $58 \%$ as the air flow rate increases by $33 \%$. The pressure drop increases slightly whenever the air flow rate increases from 5.3 to $6.6 \mathrm{~m}^{3} / \mathrm{min}$. It is recommended for this filter that the optimum filtration performance can be achieved whenever the air flow rate varies between 5.3 and $6.6 \mathrm{~m}^{3} / \mathrm{min}$. Within the previous range, it can be noticed that pressure drop increases by $8 \%$ as the air flow rate increases by $25 \%$. So, the air flow rate has a little effect on the pressure drop of a filter.

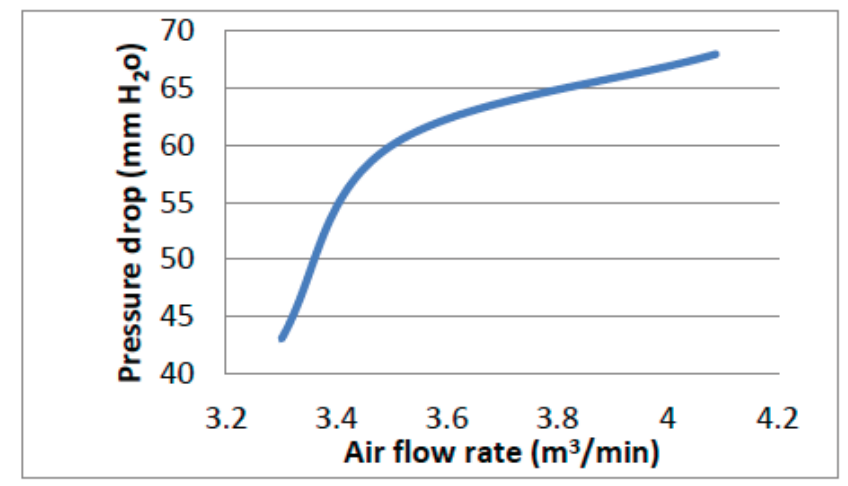

Figure 3.10. Relation between air flow rate and pressure drop for plain filter.

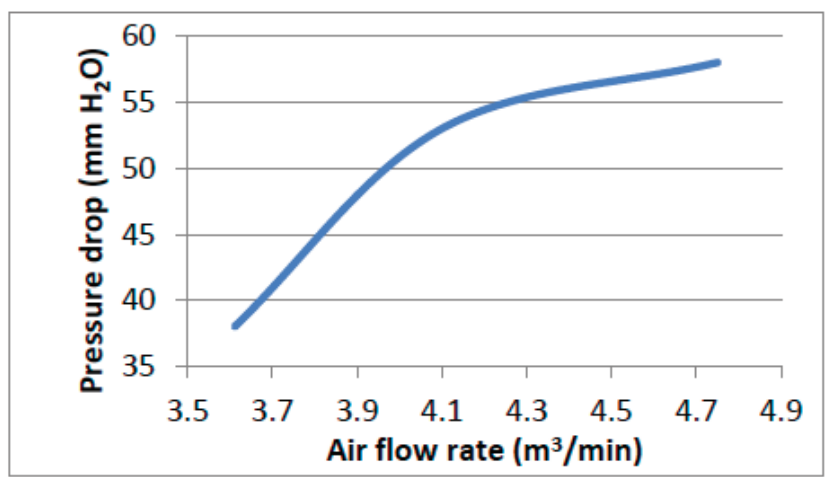

Figure 3.11 Relation between air flow rate and pressure drop for twill filter.

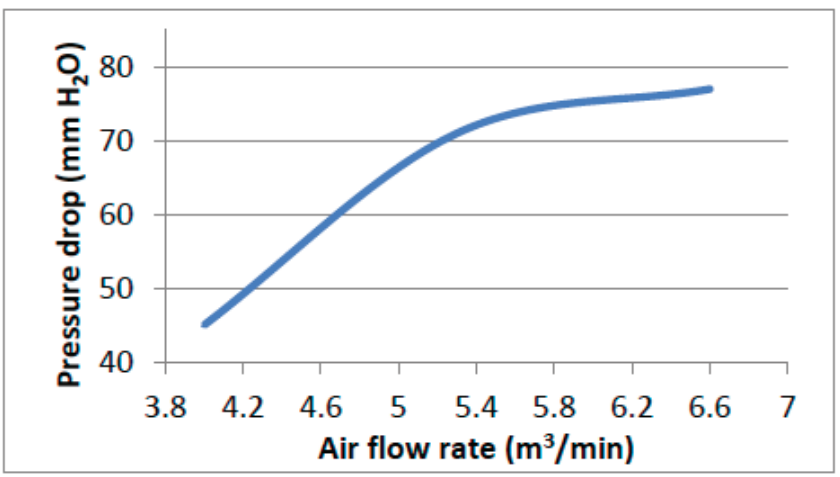

Figure 3.12. Relation between air flow rate and pressure drop for sateen filter.

Each of the three previous figures can be divided into two zones according to the slope of the line representing the relationship between air flow rate and pressure drop. The first zone is the high slope zone, while the second zone is the low slope zone. The slope of the line in the first zone is the highest one for plain filter than twill filter, while sateen filter has the least slope. It is also the same for the slope of the line in the second zone. This flow behavior assists filtration performance of sateen filters with higher air flow rates and acceptable pressure drop.

\subsubsection{Filtration efficiency and dust capacity}

Table 3.2 demonstrates that the pressure drop increases at the end of test. The increased pressure drop lets filtration efficiency to increase. The main advantage of the designed filters is the constant air flow up to the end of the test whenever the pressure drop increases. All filters introduce $100 \%$ filtration 
Table 3.2. Air filtration efficiency results.

\begin{tabular}{|c|c|c|c|c|c|c|c|c|c|}
\hline Filter & $\begin{array}{c}\text { Q start } \\
(\%)\end{array}$ & $\begin{array}{c}\Delta P \text { start } \\
(\mathrm{mm})\end{array}$ & $\begin{array}{c}Q \text { final } \\
(\%)\end{array}$ & $\begin{array}{c}\Delta P \text { final } \\
(\mathrm{mm})\end{array}$ & $M_{t}(g m)$ & $M_{\mathrm{a}}(\mathrm{gm})$ & $\eta(\%)$ & $\begin{array}{c}\text { Dust } \\
\text { concentration } \\
\left(\mathrm{gm} / \mathrm{m}^{3}\right)\end{array}$ & $M_{\mathrm{ti}}(\mathrm{gm})$ \\
\hline Plain & 100 & 50 & 100 & 72 & 24 & 0 & 100 & 3 & 588 \\
\hline Twill & 100 & 37 & 100 & 52 & 36 & 0 & 100 & 2 & 456 \\
\hline Sateen & 100 & 56 & 100 & 84 & 35 & 0 & 100 & 2 & 500 \\
\hline
\end{tabular}

$M_{t}$, dust mass on tested filter; $M_{a}$, dust mass on absolute filter; $\eta$, filtration efficiency; $M_{t i}$, total injected dust mass.

efficiency for particles greater than $25 \mathrm{~mm}$, which are suitable for air filtration applications in spinning mills, especially at the blow room area and vehicles air filters also. Dust capacities of the designed filters are approximately $4 \%$ for plain filter, $8 \%$ for twill filter and $7 \%$ for sateen filter. These low values of the dust capacities of the designed filters increase the estimated time for each filtration cycle before cleaning the filter.

\section{Conclusions}

Air passes through voids between fibers within the yarn structure and between yarns within the fabric structure. 1.8 TPI is the optimum weft twist for high permeability, but 1.4 TPI is the optimum weft twist whenever the minimum pore size is required. Increasing PPI by $50 \%$ results in decreasing air permeability by $60 \%$ to $65 \%$ and decreasing pore size by $40 \%$ to $50 \%$. Fabric thickness of sateen weave is higher than other designs; therefore, low packing density and resistance to air flow are achieved in sateen weave. For weft counts 0.8 to 1 $\mathrm{Ne}, 1.4$ to $1.8 \mathrm{TPI}$ and 16 to $20 \mathrm{PPI}$, the fabric has a reasonable range of air permeability and pore size for some filtration applications like textile industry and some vehicles motors. Controlling both yarn and fabric structures is a very important factor to achieve the designed air permeability and pore size. Nonwoven filter media are disposal materials. In addition, they cannot be cleaned completely after filtration because of the impeded particles within their structure. Finally, the designed fabric increases the competition abilities of woven filters, range of air flow rate and filtration efficiency and decreases the filtration area without scarifying efficiency.

\section{Acknowledgments}

We would like to acknowledge all staff members of FAC Filter Company at Borg Al Arab, Alexandria. Also, we wish to thank all staff members of El Nasr Wool \& Selected Textiles Company (STIA).

\section{References}

[1] Tharewal, P. G., Landage, S. M., Wasif, A. I., (2013). Application of nonwovens for air filtration. International Journal of Advanced Research in IT and Engineering, 2(2).

[2] Kolte1, P. P., Shivankar, V. S., (2015). Bilayer non-woven fabric for air filtration. International journal on Textile Engineering and Processes, 1(3).
[3] Lawrence, K., Clint, W., Wei-Yin C., (2004). Fabric filtration. Handbook of Environmental Engineering. Humana Press Inc, Totowa, NJ, Vol.1.

[4] Das, A., Alagirusamy, R., Rajan, K., (2009). Filtration characteristics of spun laced nonwoven fabric. Indian Journal of Fiber and Textile Research, 34.

[5] Irwin, H. (2007). Handbook of nonwoven filter media. Elsevier Science \& Technology Books.

[6] Kothari, V. K., Das, A., Singh, S., (2007). Filtration behavior of woven and nonwoven fabrics. Indian Journal of Fiber and Textile Research, 32.

[7] Bird, R.B., Stewart, W.E., Lightfoot, E.N., (2004). Transport phenomena, chapter 6. Aerosol Technology, Wiley \& Sons, New York.

[8] Lesson - 4 fabric filter material. (2016). Web site: https:// www.neundorfer.com.

[9] Omer Berkalp. (2006). Air permeability \& porosity in spunlaced fabrics. Fibers \& Textiles in Eastern Europe, 14(3).

[10] Elnashar, E. A. (2005). Volume porosity and permeability in double- layer woven fabrics. AUTEX Research Journal, 5(4).

[11] Ahmet C., Savvas V., Maria R. and Isik T. (2005). On the use of image processing techniques for the estimation of the porosity of textile fabrics. World Academy of Science, Engineering and Technology, 2.

[12] Jena A. and Gupta K. (2018). Characterization of pore structure of filtration media. Web site: http://www. pmiapp.com/publications/docs/Final\%20paper\%20 FluidParticle\%20Separation\%20Journal.doc.

[13] Jena A. and Gupta K. (2002). Flow porometry. Web site: $\quad$ http://www.pmiapp.com/publications/docs/Flow_ Porometry_2002.pdf.

[14] Maini, S. M., Hersh, S. P., Tucker, P. A. (1995). Barriers fabrics for protection against aerosols. Textile Progress, 26(1).

[15] Guangbiao, X. U., Fumei, W., (2005). Prediction of the permeability of woven fabrics. Journal of Industrial Textiles, 34(4).

[16] Tugrul, R. (2006). Air permeability of woven fabric. Journal of Textile and Apparel Technology and Management, 5.

[17] James, H. T. (1998). Fabric filter, Chapter 5. Research Triangle Institute.

[18] Fabric filter design variables, Lesson 3. (2018). Web site: https://www.neundorfer.com/wp-content/uploads/2016/05/ Baghouse-KnowledgeBase-03-Fabric-Filter-DesignVariables.pdf.

[19] HEPA Filters. (2018). Web site: http://www. medicalairsolutions.com/techref/hepa_filters.htm. 